\title{
Comparative effectiveness of aflibercept for the treatment of patients with neovascular age-related macular degeneration
}

This article was published in the following Dove Press journal:

Clinical Ophthalmology

7 March 2013

Number of times this article has been viewed

\author{
Michael Thomas' \\ Shaymaa S Mousa ${ }^{2}$ \\ Shaker A Mousa' \\ 'Pharmaceutical Research Institute, \\ Albany College of Pharmacy and \\ Health Sciences, Albany, NY, USA; \\ ${ }^{2}$ The Johns Hopkins University, \\ Baltimore, MD, USA
}

\begin{abstract}
Wet age-related macular degeneration (AMD) is the most common reason for vision loss in the United States. Many treatments, such as laser therapy and photodynamic therapies, have been used but their efficacy is limited. Emerging anti-vascular endothelial growth factor (VEGF) therapies are now considered the standard of care. Anti-VEGF agents inhibit angiogenesis in the eye by suppressing abnormal blood vessel growth, leading to vision improvement. Ranibizumab and bevacizumab are two examples of anti-VEGF drugs that have been approved; both showed promise based on the visual acuity scale. Aflibercept, another new therapy known to trap VEGF and inhibit multiple growth factors, is promising not only because it can be taken bimonthly based on year 1 of the VIEW trials, but it can also be extended, as demonstrated in year 2 of the VIEW trials. Based on a cost-effect analysis, aflibercept is comparable to other leading therapies. This is a review of relevant clinical trials that have proven the non-inferiority and safety of aflibercept compared to the standard of care and its unique role in the current management of wet AMD.
\end{abstract}

Keywords: aflibercept, VEGF, anti-VEGF, pegatanib, bevacizumab, ranibizumab, VIEW trials

\section{Introduction}

Wet age-related macular degeneration (AMD) is the leading cause of vision loss in adults over the age of 50 years in industrialized nations. ${ }^{1}$ Wet AMD has an increased incidence in females compared to males and is estimated to affect about 9 million people in the United States alone. ${ }^{1,2}$ As life expectancy increases and the number of senior citizens escalates, the number of people affected by wet AMD will continue to grow.

Treatment options for macular degeneration have drastically improved over the past 15 years. In the 1980s, conventional heat laser therapy was used for wet AMD, but the repercussions (scarring of the eyes, permanent vision loss) outweighed the benefits in the case of subfoveal choroidal neovascular membrane (CNVM). ${ }^{3}$ In April 2000, the US Food and Drug Administration (FDA) approved the first treatment for wet AMD: photodynamic therapy using verteporfin (Visudyne ${ }^{\circledR}$; Novartis International AG, Basel, Switzerland) injections. ${ }^{3}$ This treatment was groundbreaking at the time because of the substantial decrease of vision loss in patients with subfoveal CNVM where heat laser therapy did poorly. In photodynamic therapy, laser treatment activates verteporfin in the eye and terminates abnormal blood vessels. ${ }^{4}$ About one in six patients showed improved vision and a slowed progression of disease state with this therapy. 5,6
Correspondence: Shaker A Mousa Pharmaceutical Research Institute, Albany College of Pharmacy and Health Sciences, I Discovery Drive, Rensselaer New York 12144

Tel +I 5186947397

Fax +I 5186947567

Email shaker.mousa@acphs.edu 
Although photodynamic therapy proved to be effective, its use slowly faded because of more effective anti-vascular endothelial growth factor (VEGF) therapies. In wet AMD, VEGF promotes angiogenesis, the growth of abnormal new blood vessels in the eye. Inhibiting VEGF causes a profound reduction in vision loss. ${ }^{7}$ The first anti-VEGF breakthrough therapy used for wet AMD was pegatanib (Macugen ${ }^{\circledR}$; OSI Pharmaceuticals, Farmingdale, NY, USA/Pfizer, New York, NY, USA) followed by ranibizumab (Lucentis ${ }^{\circledR}$; Genentech Inc, South San Francisco, CA, USA; Novartis International AG), which was approved by the FDA in June $2006 .^{7,8}$ Ranibizumab is a humanized monoclonal antibody fragment that binds to the VEGF. ${ }^{9}$ Ranibizumab provided greater visual acuity benefits than photodynamic therapy.

Clinical trials have shown that bevacizumab (Avastin ${ }^{\circledR}$; Genentech Inc; Roche, Basel, Switzerland), another antiVEGF agent, is comparable in efficacy to ranibizumab, but it has not been approved for the treatment of wet AMD by the FDA as of yet. Bevacizumab showed similar efficacy compared to ranibizumab in the Comparison of AMD Treatment Trials (CATT), in which $60 \%$ of patients in both treatment groups gained visual improvements after 1 year of therapy. ${ }^{10}$ This was an eye opener for physicians because both treatments are used monthly for the treatment of wet AMD, but bevacizumab is a fraction of the cost of ranibizumab, and therefore bevacizumab is prescribed for off-label use for patients who cannot afford ranibizumab. It is preferred by the majority of ophthalmologists as first-line therapy for AMD because it was first to market, has an overall lower cost, and it has a wider spectrum of reimbursable indications.

The most recently approved treatment for wet AMD is aflibercept $\left(\right.$ Eylea $\left.^{\circledR}\right),{ }^{11}$ co-developed by Sanofi-Aventis (Paris, France) and Regeneron Pharmaceuticals (Tarrytown, NY, USA) and approved by the FDA in November 2011. It works by binding tightly to three isoforms of growth factors (VEGFA, VEGF-B, and placental growth factor). ${ }^{12}$ This agent has high binding affinity and a long half-life, which makes afibercept lucrative in terms of the possibility of cost savings and decreased frequency of use. This review will compare the effectiveness of aflibercept versus current therapies based on clinical trials for patients with neovascular (wet) AMD.

\section{Methodology}

Using databases such as PubMed, Medline, and ClinicalTrials.gov, data were collected to compare the efficacy of aflibercept versus other therapies in the treatment of wet AMD. Data were also collected from online sources such as the National Eye Institute, ${ }^{13}$ Macular Degeneration
Association, ${ }^{2}$ other drug-dosing information webpages, and peer-review journals in order to give a fuller description of the pharmaceutical agents and their respective strengths. ${ }^{14,15}$

\section{Wet AMD pathophysiology}

To fully understand macular degeneration the key components that are affected need to be defined. The macula is responsible for fine tuning images within one's visual field, and impairment of this function can lead to ensuing vision loss. ${ }^{16}$ Macular degeneration can be broken down into two subsets: dry (nonexudative) AMD and wet (exudative) AMD. ${ }^{17}$ Dry AMD occurs in about $85 \%$ of patients diagnosed with macular degeneration. ${ }^{12}$ The less serious of the two, dry AMD, is defined by drusen, small yellow deposits located near the retina. ${ }^{18}$ Dry AMD is categorized into stages based on the size of the drusen, the degree of vision loss, and retinal pigment epithelium hyperpigmentation and atrophy. ${ }^{18}$

Wet AMD accounts for only $15 \%$ of all macular degeneration, but causes up to $90 \%$ of blindness in individuals diagnosed with AMD. ${ }^{19}$ Individuals with wet AMD have weak blood vessels underneath the retina and macula, leading to the leakage of fluids and blood into the eye and consequently causing macular damage. ${ }^{2,20}$ Cytokines and other inflammatory markers such as VEGF cause ischemia and inflammation, which leads to choroidal neovascularization (CNV). ${ }^{2}$ In CNV, new blood vessels grow into the subretinal pigment epithelium (type 1) or subretinal space (type 2) by breaking through the Bruch membrane, therefore leading to rapid vision loss of central vision and metamorphopsia on Amsler grid testing. ${ }^{16}$

\section{VEGF and its role in wet AMD}

VEGF, which occurs naturally in the body, works by stimulating chemical signals to promote the growth of new blood vessels in areas of the body that are oxygen deficient. ${ }^{21}$ It does so by binding to a tyrosine kinase receptor, causing activation by phosphorylation (Figure 1). The VEGF receptor (VEGFR) consists of seven domains, and almost all activation occurs by binding to VEGFR-1 and VEGFR-2 domains. ${ }^{22}$ Both VEGFR-1 and VEGFR-2 are located in the vascular endothelium, neurosensory retina, and retinal pigment epithelium cells. ${ }^{22}$ Activation of these receptors is the focal point of angiogenesis and vascular growth where the role of VEGFR-2, but not VEGFR-1, is documented. In other disease states, especially in cancer and tumor growth, overexpression of VEGF can lead to increased blood vessel growth, thus aiding tumor growth by providing an adequate oxygen supply. 


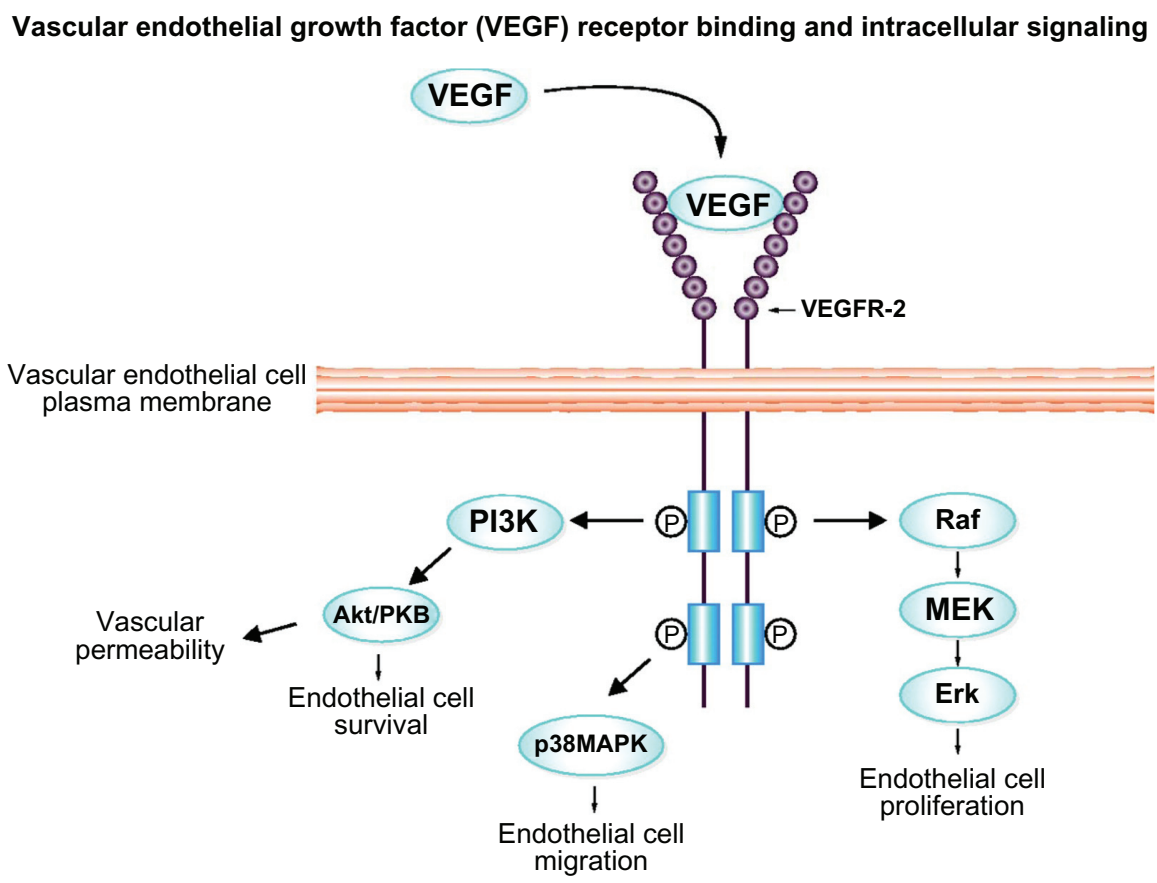

Figure I VEGF binds to the VEGFR-2 receptor, activating angiogenic response by phosphorylating domains within the receptor and below the endothelial membrane. Note: Reprinted with permission. (C) 2005 American Society of Clinical Oncology. All rights reserved. From: Rini BI, Small EJ. Biology and clinical development of vascular endothelial growth factor-targeted therapy in renal cell carcinoma. J Clin Oncol. 2005;23(5): I028-1043.50

Abbreviations: VEGF, vascular endothelial growth factor; VEGFR, VEGF receptor.

Wet AMD is illustrated by the development of CNV. In CNV the innermost layer, the Bruch's membrane, becomes inflamed and causes thickening of the vascular endothelium. ${ }^{23,24}$ Irregular growth of blood vessels under the macula supports this thickening and causes rapid central vision loss. Even in advanced stages of wet AMD partial vision is still retained, and severe loss of peripheral vision is uncommon. ${ }^{25}$ The thickening and addition of new blood vessels leads to inflammation of the macula and eventual scarring of the retina and macula. ${ }^{26}$ The vessels are undeveloped and cause a leakage of fluids into the eye, leading to inflammation.

$\mathrm{CNV}$ has a strong link to the increased expression of the $V E G F$ gene. ${ }^{27}$ VEGF is often considered the main growth factor leading to the increased angiogenesis within the eye. VEGF maintains this vessel growth, along with other cytokines such as tumor necrosis factor-alpha and a host of interleukins. ${ }^{8}$ In wet AMD, inflammatory events that cause damage to the eye happen at a much faster rate than in dry AMD. Additionally, oxidative stress, complement factor $\mathrm{H}$ gene, and lifestyle factors such as diet and smoking might play a role in the etiology of AMD. ${ }^{28,29}$

\section{Current management of wet AMD}

Wet AMD treatment options continue to expand, with new and more effective agents. Photodynamic therapy is no longer recommended as a monotherapy regimen, but success has been shown in combination with pharmacological treatment. ${ }^{30}$ Currently, the standard of care for wet AMD is either a monthly dose of bevacizumab or ranibizumab. ${ }^{31}$ Ranibizumab shows promising results in patients with wet AMD, improving vision in over one-third of the patients tested. ${ }^{32}$ It has relatively few side effects, such as eye irritation and redness, most of which are due to the injection itself. ${ }^{33}$ Bevacizumab has shown non-inferior results when compared to ranibizumab in the CATTs. ${ }^{10}$ Many physicians tend to use this medication off-label due to its cost effectiveness versus ranibizumab.

\section{Aflibercept}

Aflibercept, unlike other VEGF inhibitors, is a recombinant protein in which VEGFR-1 (second binding domain) and VEGFR-2 (third binding domain) are attached to the Fc portion of human immunoglobulin G. ${ }^{34}$ Aflibercept differs from ranibizumab and bevacizumab in the mode of action by targeting VEGF via acting as a dummy receptor for VEGF, thus effectively inhibiting the angiogenic response. ${ }^{35}$ This targeted response traps VEGF by binding to it even more tightly than its native receptor, triggering no angiogenic action from the VEGF and leaving it inactive. ${ }^{12}$ Without the cascade of angiogenic responses, fewer leaky vessels grow 
into the macula. Aflibercept slows the progression of wet AMD, allowing patients to retain central vision for much longer.

VEGF is categorized into VEGF-A, VEGF-B, VEGF-C, VEGF-D, VEGF-E, and placenta growth factor (PIGF). ${ }^{14,35}$ Aflibercept, a fusion protein with binding domains from native VEGF receptors, binds with high affinity to VEGF-A, VEGF-B, PIGF1, and PIGF2. ${ }^{14}$ Preclinical studies demonstrated that aflibercept suppressed CNV in several animal models. ${ }^{34}$ The result of Phase III trials in humans showed effective short-term suppression of CNV in patients with exudative AMD and suggested longer durability of aflibercept compared with ranibizumab and bevacizumab. ${ }^{36,37}$ A pivotal Phase III VEGF trial in patients with wet AMD showed that aflibercept was noninferior to ranibizumab in preventing vision loss with comparable vision gains, safety, and perhaps at lower cost than ranibizumab. ${ }^{35}$

\section{Pharmacokinetics}

Aflibercept has the highest affinity for VEGF165 ( $\left.\mathrm{K}_{\mathrm{d}} 0.49 \mathrm{Pm}\right)$ compared to ranibizumab $\left(\mathrm{K}_{\mathrm{d}} 46 \mathrm{Pm}\right)$ and bevacizumab $\left(\mathrm{K}_{\mathrm{d}} 58 \mathrm{Pm}\right) .{ }^{19,37}$ Moreover, aflibercept is the only approved therapy for exudative AMD that inhibits VEGF-B and PIGF, giving it further claim to be a promising solution for wet AMD. Using aflibercept can potentially inhibit more angiogenic factors, possibly treating wet AMD more effectively than current therapies.

The most captivating aspect of aflibercept is its extended half-life. According to reviews of aflibercept by Stewart ${ }^{8}$ and Stewart et al, ${ }^{35}$ the estimated serum half-life of intravitreal aflibercept is approximately 18 days versus ranibizumab and bevacizumab, which have half-lives of approximately 4.75 days and 8.25 days, respectively. Stewart et al estimated intravitreal half-life for aflibercept to be 7.1 days. ${ }^{35}$ The reason for aflibercept's unusually long half-life is due to its relatively large molecular weight of $115 \mathrm{Kd}$, which allows it to circulate within the eye for a longer time. ${ }^{22}$ Owing to its prolonged half-life, bimonthly dosing is possible and has altered current regimens of monthly dosing for exudative AMD. Dosing in such a way can provide immediate benefits to patients who have a difficult time with intravitreal dosage administration. Also with the extended dosing intervals, patients are less likely to face side effects from the dosage regimen and are less likely to have medication administration errors.

\section{Clinical evidence}

Aflibercept gained FDA approval after two randomized, double-blind Phase III trials were conducted: VIEW 1 and
VIEW 2 (VEGF Trap-Eye: Investigation of Efficacy and Safety in Wet Age-Related Macular Degeneration). ${ }^{36,37}$ These studies were done to measure the safety and efficacy of aflibercept compared to the current standard of care for wet AMD, ranibizumab. It is important to note that the VIEW studies were run concurrently in different parts of the world and both studies had the same endpoints, treatment group population, and primary outcome measures. The only difference was that VIEW 1 was conducted in North America, whereas VIEW 2 was conducted internationally. Both trials had a set outcome goal at 52 weeks of treatment and the primary outcome was identified as the percentage of patients who maintained vision at week 52. Maintaining vision was defined as losing less than 15 letters based on the best-corrected visual acuity scale compared to baseline measurements. ${ }^{36}$ There were four treatment groups into which patients were randomly placed (Table 1). ${ }^{36,37}$

The results in all four treatment groups yielded similar conclusions. The primary endpoint showed noninferiority in all four treatment groups where noninferiority was defined in comparison to the standard of care ranibizumab and concluding that the three aflibercept groups were noninferior. ${ }^{36,37}$ The most pertinent data for the use of aflibercept show the success of aflibercept dosed every 8 weeks. In year 2 of the VIEW trials, when dosing was switched to as-needed, aflibercept again proved noninferior to ranibizumab. ${ }^{37}$ The safety analysis in both VIEW trials found aflibercept to be a well-tolerated drug. ${ }^{38}$ When compared to ranibizumab, the safety profile was of approximately equivalent measures. ${ }^{36,39}$ In conclusion, aflibercept dosed intravitreally each month or every 2 months after three initial monthly doses resulted in comparable efficacy and safety to monthly ranibizumab. Side effects, either ocular or systemic, were similar across treatment groups, with no differences between aflibercept administered every 2 months and monthly ranibizumab. ${ }^{38}$

These studies provided a foundation for using aflibercept therapy in wet AMD. Potential advantages of aflibercept

Table I Treatment groups in the VIEW I and VIEW 2 clinical trials

\begin{tabular}{lll}
\hline $\begin{array}{l}\text { Drug } \\
\text { (intravitreal } \\
\text { injection) }\end{array}$ & $\begin{array}{l}\text { Dosage } \\
(\mathbf{m g})\end{array}$ & $\begin{array}{l}\text { Dosing frequency }(\mathbf{n}) \\
\text { (every } \mathbf{n} \text { weeks for } \\
\mathbf{5 2} \text { weeks, then as needed) }\end{array}$ \\
\hline Ranibizumab & 0.5 & 4 \\
Aflibercept & 0.5 & 4 \\
Aflibercept & 2 & 4 \\
Aflibercept & 2 & 8 \\
\hline
\end{tabular}

Abbreviation: VIEW, VEGF Trap-Eye: Investigation of Efficacy and Safety in Wet Age-Related Macular Degeneration. 
might include extended dosing periods that might lead to fewer hospital and physician visits and less financial cost to the patient due to less frequent dosing.

\section{Cost-effect analysis}

The recommended dose for aflibercept is $2 \mathrm{mg}$ every 4 weeks for 3 months, followed by $2 \mathrm{mg}$ every 8 weeks. ${ }^{40}$ More frequent dosing was not found to be advantageous to patients. ${ }^{41}$ Ranibizumab is currently dosed at $0.5 \mathrm{mg}$ monthly as per guidelines. ${ }^{9}$ Although there are only three FDA-approved therapies for wet AMD, it should also be noted that bevacizumab is the most-used therapy for wet AMD because of its relatively low cost and comparable efficacy to ranibizumab. Bevacizumab is most often dosed at $1.25-2.5 \mathrm{mg}$ per monthly treatment for macular degeneration. ${ }^{42}$ All three therapies are considered to have equal efficacy across the board based on studies and clinical data that have been evaluated. ${ }^{31}$

For one treatment of ranibizumab, the cost of medication totals USD1950 per vial/dose. ${ }^{43}$ If a patient is on ranibizumab for 1 year, the cost of therapy is USD23,400 (12 doses). ${ }^{44}$ For aflibercept, due to the less intensive dosing regimen, clear savings can be seen. A single dose/treatment of aflibercept costs approximately USD1850 and the yearly cost averages USD14,800 (eight doses). Bevacizumab is the cheapest, costing roughly USD50 per dose/treatment. A yearly treatment of one bevacizumab dose per month would cost about USD600. ${ }^{45,46}$ Not included in these cost analyses is the cost of treatment (physician visits, optical coherence tomography costs, and injection costs), which varies from center to center and is not readily available.

\section{Other indications for aflibercept}

Experiments to use intravitreal aflibercept for the treatment of diabetic macular edema have shown promising results in clinical trials..$^{47-49}$ The results of a Phase II clinical trial showed that anti-VEGF therapies improved vision compared to the standard of care for diabetic macular edema, which is photocoagulation therapy.

\section{Conclusion}

It is obvious that aflibercept has a role in macular degeneration therapy, but its use is currently limited. The major limiting factor is the cost of the therapy and administration. With bevacizumab already being the most-used anti-VEGF drug for macular degeneration, even though it is being used off-label, its use is not anticipated to subside. The CATT research group found the monthly use of either bevacizumab or ranibizumab results in the same visual acuity outcome. ${ }^{10}$ Such comparisons have raised questions about which drug to choose. Physicians tend to prescribe bevacizumab due to its extremely low cost compared to aflibercept and ranibizumab.

Aflibercept is an intriguing choice for patients who have a difficult time with the intravitreal injections. After the initial three loading doses, bimonthly dosing can be beneficial for noncompliant patients. Compared to current treatments, aflibercept has shown equal efficacy and safety. Its unique pharmacokinetics and binding to multiple receptors may allow it to expand its role in other disease states as well.

\section{Disclosure}

The authors report no conflicts of interest in this work.

\section{References}

1. Haddrill M. Macular degeneration treatment [webpage on the Internet]. San Diego, CA: Access Media Group LLC; 2011 [updated Nov 2011; cited Oct 2012]. Available from: http://www.allaboutvision.com/ conditions/amd-treatments.htm. Accessed October 23, 2012.

2. About macular degeneration [webpage on the Internet]. Sarasota, FL: Macular Degeneration Association; 2011 [cited October 26, 2012]. Available from: http://www.maculardegenerationassociation.org/ about-md/. Accessed February 25, 2013.

3. MedlinePlus: Verteporfin injection [webpage on the Internet]. Bethesda, MD: US National Library of Medicine [updated September 1, 2010]. Available from: http://www.nlm.nih.gov/medlineplus/druginfo/meds/ a607060.html. Accessed February 25, 2013.

4. Ahn JC, Chung PS. The activity of G-ROS and the predominant role of Type II reaction in the photodynamic therapy using 9-hydroxypheophorbide-alpha for HeLa cell lines. Gen Physiol Biophys. 2012;31(3):343-350.

5. Yoo JJ, Kim C, Chung CW, Jeong YI, Kang DH. 5-aminolevulinic acid-incorporated poly(vinyl alcohol) nanofiber-coated metal stent for application in photodynamic therapy. Int J Nanomedicine. 2012;7: 1997-2005.

6. Treatment techniques and clinical guidelines for photocoagulation of diabetic macular edema. Early Treatment Diabetic Retinopathy Study Report Number 2. Early Treatment Diabetic Retinopathy Study Research Group. Ophthalmology. 1987;94(7):761-774.

7. Chen Y, Han F. Profile of ranibizumab: efficacy and safety for the treatment of wet age-related macular degeneration. Ther Clin Risk Manag. 2012;8:343-351.

8. Stewart MW. Clinical and differential utility of VEGF inhibitors in wet age-related macular degeneration: focus on aflibercept. Clin Ophthalmol. 2012;6:1175-1186.

9. Chen E, Brown DM, Wong TP, et al. Lucentis using Visudyne study: determining the threshold-dose fluence of verteporfin photodynamic therapy combined with intravitreal ranibizumab for exudative macular degeneration. Clin Ophthalmol. 2010;4:1073-1079.

10. CATT Research Group, Martin DF, Maguire MG, et al. Ranibizumab and bevacizumab for neovascular age-related macular degeneration. N Engl J Med. 2011;364(20):1897-1908.

11. Aflibercept (Eylea) for age-related macular degeneration [webpage on the Internet]. New Rochelle, NY: The Medical Letter, Inc; 2012 [updated February 6, 2012]. Available from: http://secure.medicalletter. org/TML-article-1383a. Accessed November 13, 2012.

12. Browning DJ, Kaiser PK, Rosenfeld PJ, Stewart MW. Aflibercept for age-related macular degeneration: a game-changer or quiet addition? Am J Ophthalmol. 2012;154(2):222-226. 
13. NEI: National Eye Institute [homepage on the Internet]. Bethesda, MD: National Institutes of Health [updated Sep 2009]. Available from: http:// www.nei.nih.gov/. Accessed November 13, 2012.

14. Stewart MW. The expanding role of vascular endothelial growth factor inhibitors in ophthalmology. Mayo Clin Proc. 2012;87(1):77-88.

15. Chong V. Biological, preclinical and clinical characteristics of inhibitors of vascular endothelial growth factors. Ophthalmologica. 2012; 227 Suppl 1:2-10.

16. Facts about age-related macular degeneration [webpage on the Internet]. Bethesda, MD: National Institutes of Health; 2009 [updated Sep 2009]. Available from: http://www.nei.nih.gov/health/maculardegen/armd_ facts.asp. Accessed November 13, 2012.

17. Campochiaro PA. Anti-vascular endothelial growth factor treatment for retinal vein occlusions. Ophthalmologica. 2012;227 Suppl 1:30-35.

18. Costagliola C, Agnifili L, Arcidiacono B, Duse S, Fasanella V, Mastropasqua $\mathrm{R}$, et al. Systemic thromboembolic adverse events in patients treated with intravitreal anti-VEGF drugs for neovascular age-related macular degeneration. Expert Opin Biol Ther. 2012;12(10): 1299-1313.

19. Christoforidis JB, Williams MM, Kothandaraman S, Kumar K, Epitropoulos FJ, Knopp MV. Pharmacokinetic properties of intravitreal I-124-aflibercept in a rabbit model using PET/CT. Curr Eye Res. 2012;37(12):1171-1174.

20. Golik P, Tońska K. Comparison of the biological principles underlying the action of monoclonal antibody $(\mathrm{mAb})$ and decoy receptor antiVEGF agents - on the example of ranibizumab (anti-VEGF-A mAb) and aflibercept (decoy VEGFR1-2 receptor). Klin Oczna. 2012;114(1): 79-83. Polish.

21. Papadopoulos N, Martin J, Ruan Q, et al. Binding and neutralization of vascular endothelial growth factor (VEGF) and related ligands by VEGF Trap, ranibizumab and bevacizumab. Angiogenesis. 2012;15(2): 171-185.

22. Verner-Cole EA, Davis SJ, Lauer AK. Aflibercept for the treatment of neovascular age-related macular degeneration. Drugs Today (Barc). 2012;48(5):317-329.

23. Veritti D, Sarao V, Lanzetta P. Neovascular age-related macular degeneration. Ophthalmologica. 2012;227 Suppl 1:11-20.

24. Ohr M, Kaiser PK. Intravitreal aflibercept injection for neovascular (wet) age-related macular degeneration. Expert Opin Pharmacother. 2012;13(4):585-591.

25. Nork TM, Dubielzig RR, Christian BJ, et al. Prevention of experimental choroidal neovascularization and resolution of active lesions by VEGF trap in nonhuman primates. Arch Ophthalmol. 2011;129(8): 1042-1052.

26. Ida H, Tobe T, Nambu H, Matsumura M, Uyama M, Campochiaro PA. RPE cells modulate subretinal neovascularization, but do not cause regression in mice with sustained expression of VEGF. Invest Ophthalmol Vis Sci. 2003;44(12):5430-5437.

27. Nagineni CN, Kommineni VK, William A, Detrick B, Hooks JJ. Regulation of VEGF expression in human retinal cells by cytokines: implications for the role of inflammation in age-related macular degeneration. J Cell Physiol. 2012;227(1):116-126.

28. Jarrett SG, Boulton ME. Consequences of oxidative stress in age-related macular degeneration. Mol Aspects Med. 2012;33(4):399-417.

29. Sparrow JR, Ueda K, Zhou J. Complement dysregulation in AMD: RPE-Bruch's membrane-choroid. Mol Aspects Med. 2012;33(4): 436-445.

30. Couch SM, Bakri SJ. Review of combination therapies for neovascular age-related macular degeneration. Semin Ophthalmol. 2011;26(3): 114-120.

31. Kovach JL, Schwartz SG, Flynn HW Jr, Scott IU. Anti-VEGF treatment strategies for wet AMD. J Ophthalmol. 2012;2012:786870.

32. Meyer CH, Holz FG. Preclinical aspects of anti-VEGF agents for the treatment of wet AMD: ranibizumab and bevacizumab. Eye (Lond). 2011;25(6):661-672.

33. Figurska M, Robaszkiewicz J, Wierzbowska J. Safety of ranibizumab therapy in wet AMD and the role of vascular endothelial growth factors in physiological angiogenesis. Klin Oczna. 2010;112(4-6):147-150.
34. Traynor K. Aflibercept approved for macular degeneration. Am J Health Syst Pharm. 2012;69(1):6.

35. Stewart MW, Grippon S, Kirkpatrick P. Aflibercept. Nat Rev Drug Discov. 2012;11(4):269-270.

36. Regeneron Pharmaceuticals. Vascular Endothelial Growth Factor (VEGF) Trap-Eye: Investigation of Efficacy and Safety in Wet AgeRelated Macular Degeneration (AMD) (VIEW 1). In: ClinicalTrials. gov [website on the Internet]. Bethesda, MD: US National Library of Medicine; 2007 [updated December 20, 2012]. Available from: http://clinicaltrials.gov/ct2/show/NCT00509795. NLM identifer: NCT00509795. Accessed November 13, 2012.

37. Bayer. Vascular Endothelial Growth Factor (VEGF) Trap-Eye: Investigation of Efficacy and Safety in Wet Age-Related Macular Degeneration (AMD) (VIEW 2) In: ClinicalTrials.gov [website on the Internet]. Bethesda, MD: US National Library of Medicine; 2008 [updated February 27, 2012]. Available from: http://clinicaltrials.gov/ct2/show/ NCT00637377. NLM identifer: NCT00637377. Accessed November 13, 2012.

38. Heier JS, Brown DM, Chong V, et al; VIEW 1 and VIEW 2 Study Groups. Intravitreal aflibercept (VEGF Trap-Eye) in wet age-related macular degeneration. Ophthalmology. 2012;119(12):2537-2548.

39. Frampton JE. Aflibercept for intravitreal injection : in neovascular agerelated macular degeneration. Drugs Aging. 2012;29(10):839-846.

40. Nguyen QD, Campochiaro PA, Shah SM, et al; Clear-It 1 Investigators. Evaluation of very high- and very low-dose intravitreal aflibercept in patients with neovascular age-related macular degeneration. $J$ Ocul Pharmacol Ther. 2012;28(6):581-588.

41. Stewart MW, Rosenfeld PJ, Penha FM, et al. Pharmacokinetic rationale for dosing every 2 weeks versus 4 weeks with intravitreal ranibizumab, bevacizumab, and aflibercept (vascular endothelial growth factor Trap-eye). Retina. 2012;32(3):434-457.

42. Costa RA, Jorge R, Calucci D, Cardillo JA, Melo LA Jr, Scott IU. Intravitreal bevacizumab for choroidal neovascularization caused by AMD (IbeNA Study): results of a phase 1 dose-escalation study. Invest Ophthalmol Vis Sci. 2006;47(10):4569-4578.

43. Jackson TL, Kirkpatrick L. Cost comparison of ranibizumab and bevacizumab. BMJ. 2011;343:d5058.

44. Hurley SF, Matthews JP, Guymer RH. Cost-effectiveness of ranibizumab for neovascular age-related macular degeneration. Cost Eff Resour Alloc. 2008;6:12.

45. Mitchell P, Annemans L, Gallagher M, et al. Cost-effectiveness of ranibizumab in treatment of diabetic macular oedema (DME) causing visual impairment: evidence from the RESTORE trial. Br J Ophthalmol. 2012;96(5):688-693.

46. Raftery J, Clegg A, Jones J, Tan SC, Lotery A. Ranibizumab (Lucentis) versus bevacizumab (Avastin): modelling cost effectiveness. $\mathrm{Br} \mathrm{J}$ Ophthalmol. 2007;91(9):1244-1246.

47. Lang GE. Diabetic macular edema. Ophthalmologica. 2012;227 Suppl 1: 21-29.

48. Bandello F, Berchicci L, La Spina C, Battaglia Parodi M, Iacono P. Evidence for anti-VEGF treatment of diabetic macular edema. Ophthalmic Res. 2012;48 Suppl 1:16-20.

49. Diabetic Retinopathy Clinical Research Network. Comparative Effectiveness Study of Intravitreal Aflibercept, Bevacizumab, and Ranibizumab for DME (Protocol T). In: ClinicalTrials.gov [website on the Internet]. Bethesda, MD: US National Library of Medicine; 2012 [updated August 21, 2012]. Available from: http://clinicaltrials.gov/ ct2/show/NCT01627249. NLM identifier: NCT01627249. Accessed November 14, 2012.

50. Rini BI, Small EJ. Biology and clinical development of vascular endothelial growth factor-targeted therapy in renal cell carcinoma. J Clin Oncol. 2005;23(5):1028-1043. 
Clinical Ophthalmology

\section{Publish your work in this journal}

Clinical Ophthalmology is an international, peer-reviewed journal covering all subspecialties within ophthalmology. Key topics include: Optometry; Visual science; Pharmacology and drug therapy in eye diseases; Basic Sciences; Primary and Secondary eye care; Patien Safety and Quality of Care Improvements. This journal is indexed on

PubMed Central and CAS, and is the official journal of The Society of Clinical Ophthalmology (SCO). The manuscript management system is completely online and includes a very quick and fair peer-review system, which is all easy to use. Visit http://www.dovepress.com/ testimonials.php to read real quotes from published authors. 